D. COUSIN, S. FORTIER

ARCADIS ESG 18, rue Troyon 92316 Sèvres

\title{
Confortement des fondations de deux bâtiments sinistrés par la sécheresse à Palaiseau Reprise-en sous-oeuvre des fondations par micropieux et longrines
}

Les immeubles D et $E$ du Parc d'Ardenay, implantés sur le flanc de la colline de Palaiseau, ont subi d'importants désordres à la suite de la sécheresse et de mouvements de terrains. ARCADIS a élaboré un dispositif de confortement consistant en une rigidification en béton armé de la structure par longrines, associée à une reprise en sous-ceuve des fondations par micropieux. Larticle présente les aspects conception et réalisation de ce chantier de confortement qui dolt s'achever en maj 2007.

Mots-clés : mouvement de terrains, sécheresse, confortement, reprise en sous-ceuvre, micropieux, longrines.

\section{Confortement of two buildings foun- dations' victim by the dryness at Palaiseau \\ Underpinning of the foundations by micropiles and footings}

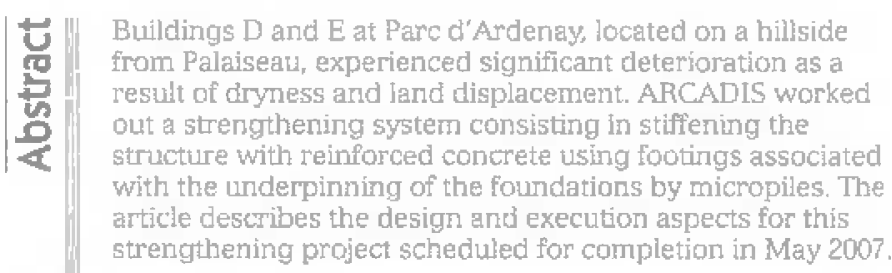

Keywords: and displacement, dryness, strengthening. underpinning, micropile, footing. 
Les immeubles D et E, rue d'Ardenay à Palaiseau, sont situés sur le flanc de coteau sud-est du plateau de Palajseau dominant toute la vallée de la Biève au nord et de l'Yvette au sud. Ces bâtiments font partie d'un ensemble de six bâtiments réalisés dans tes années 1970 en pérìphérie du château d'Ardenay dans le parc d'Arçenay.

D'orientation principale $\mathrm{E}-\mathrm{O}$ (Fig. 1), les bâtiments $\mathrm{D}$ et $\mathrm{E}$, de type $\mathrm{R}+3 / 5$ avec sous-sol sermi enterré usage de caves, sont bordés en façade nord par la rue d'Ardenay et en façade sud par le coteau de Palaiseau. IIs sont situés vers la cote de $+145 \mathrm{NGE}$, toutefois le bâtiment E suit la pente du talus pour atteindre, en partie aval, la cote de $+140 \mathrm{NGF}$.
Les dimensions des bâtiments D et E sont respectivement de $70 \mathrm{~m}$ et $130 \mathrm{~m}$ pour la longueur et de I'ordre de $8 \mathrm{~m}$ pour la largeur (Fig. 1 et 2).

A l'exception des façades de type voile, la structure des immeubles est constituée de poteaux et poutres en béton armé. Les fondations des immeubles sont superficielles, de type semelles isolées et filantes ; elles reposent vers $0,80 \mathrm{~m}$ de profondeur par rapport au niveau bas du sous-sol et vers 2 m de profondeur par rapport au niveau du terrain naturel, dans les argiles à meuliêre en place.

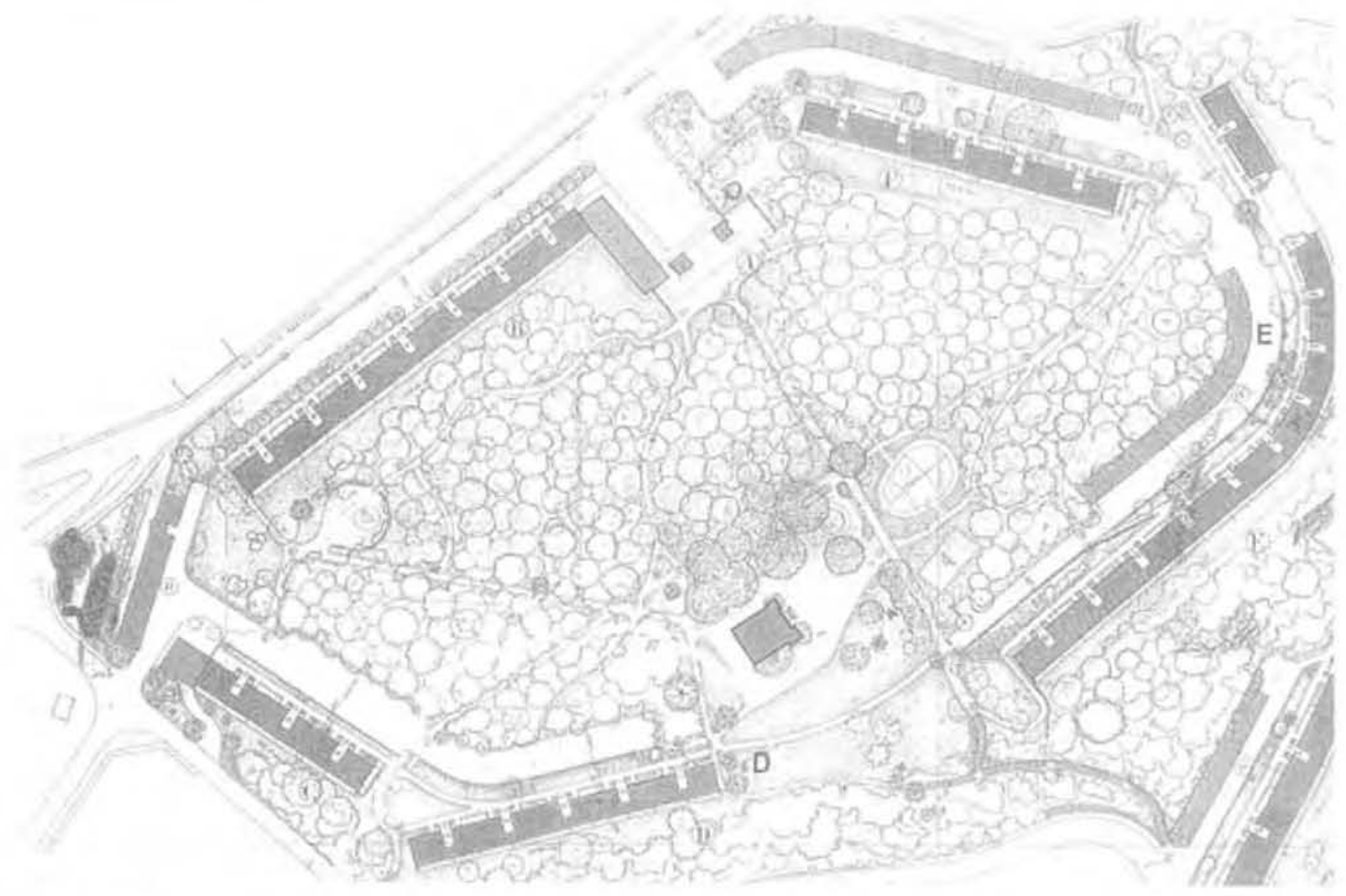

Fis. 1 Plan masse des bâtiments $\mathrm{D}$ et $\mathrm{E}$.

Plan of buildings $\mathrm{D}$ and $\mathrm{E}$.
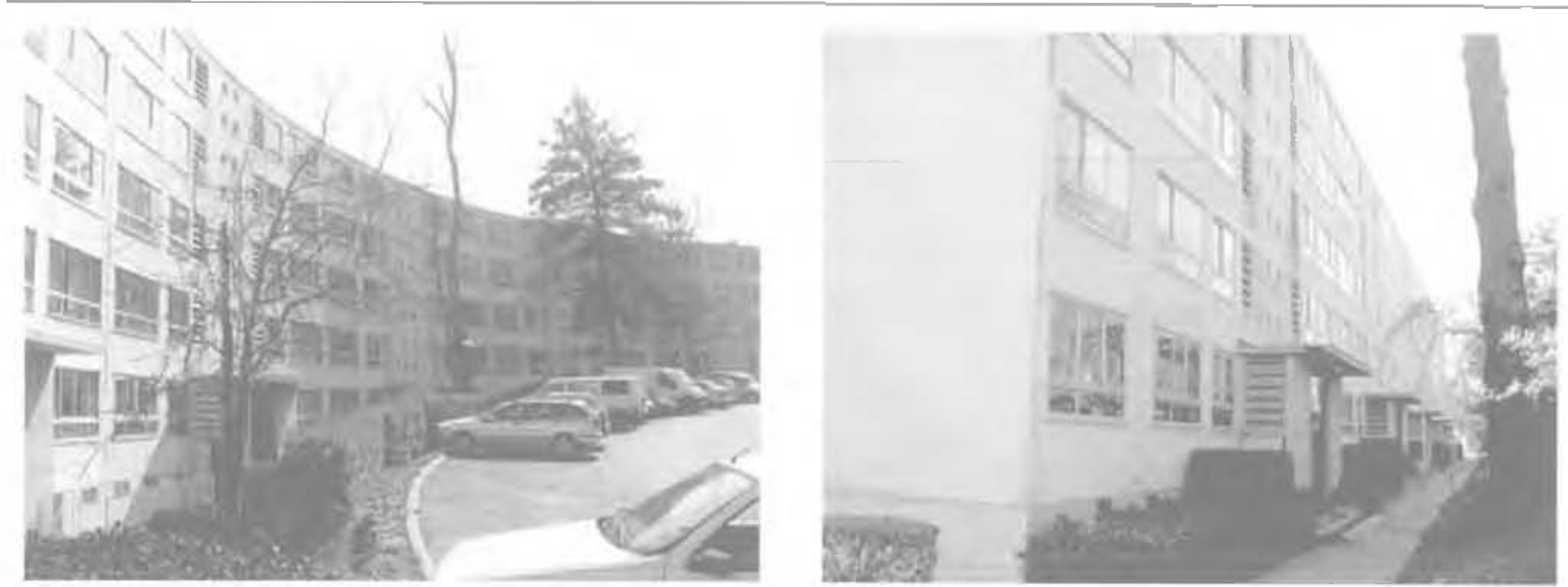

FG. ㄴ. Vues des bâtiments E et D.

View of buildings $\mathrm{E}$ and $\mathrm{D}$. 


\section{Géologie}

Du point de vue géologique, on rencontre dans cette zone, d'après la carte géologique de Corbeil au 1/50 000 , des résidus locaux de limons des plateaux, puis les argiles à meulière sur 3 à $4 \mathrm{~m}$ d'épaisseur sumontant les sables de Fontainebleau (Fig. 3). Une nappe d'eau superficielle alimentée par les eaux météoriques peut baigner les limons des plateaux, retenue par les argiles à meulière peu pernéables. Très fluants, les argiles à meulière et les sables de Fontainebleau de nature argileuse recouvrent généralement les niveaux sousjacents des marnes à huîtres el du marno-calcaire de Brie sur le pied de la colline.

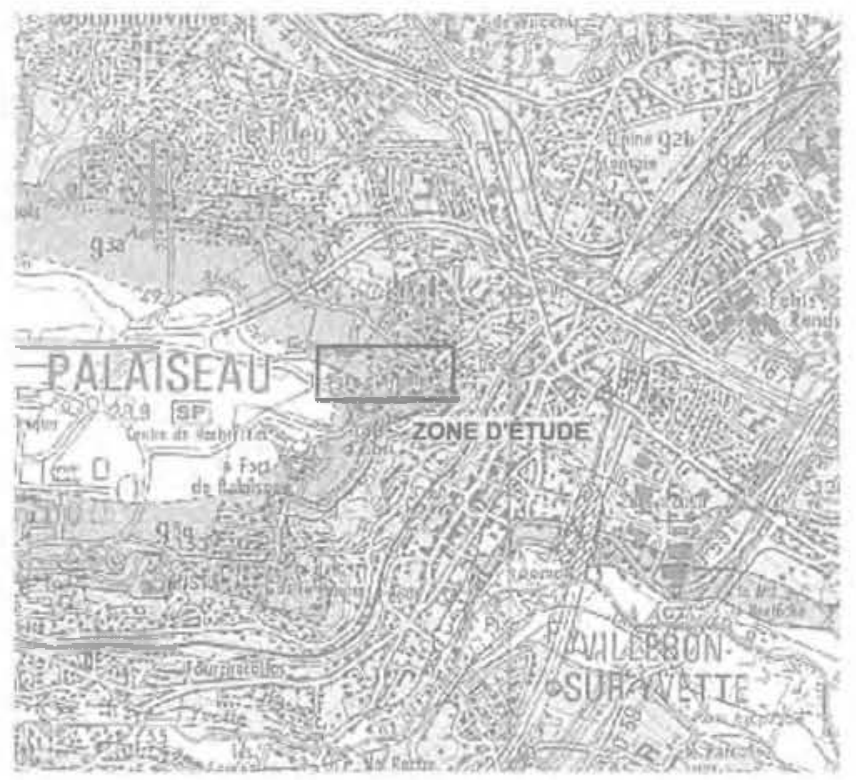

คG. 3 Extrait de la carte géologique de Corbeil au 1/50 000 (BRGM, 1979).

Extract of the geological map of Corbeil.

\section{2}

\section{Les désordres}

Sur ces deux immeubles, les désordres se traduisent par l'apparition de fissures intérieures et traversantes au droit des planchers haut du rez-de-chaussée, prenant naissance à partir des poteaux ainsi que d'autres fissures localisces sur les murs cloisons accolés aux poteaux. Les poteaux intérieurs sont affectés par un cisaillement important aux líaisons avec les murs intérieurs. A l'extérieur, des fissures verticales louverture de l'ordre du centimètre) prolongent les joints de dilatation sur la hauleur des voiles des facades des immeubles. Dans les caves, le dallage est fortement fissuré et d'autres fissures affectent les murs cloisons des caves.

Ce sinistre a fait l'objet d'une expertise judiciaire durant laquelle ARCADIS ESG a réakisé un diagnostic géotechnique afin de déterminer l'origine des désordres et de proposer une solution de travaux confortatifs pour mettre en sécurité les deux bátiments. Eri complément, une instrumentation a été mise en place sur les båtiments sinistrés pour permettre de suivre au fil des saisons, l'évolution des désordres. Cette irs- trumentation a comporté la pose d'extensomètres tridirectionnels (mesure de l'écartement, du rejet et du glissement) sur les fissures et les joints, et de nivelles sur les façades (mesure de l'inclinaison) (Fig. 4 et 5).

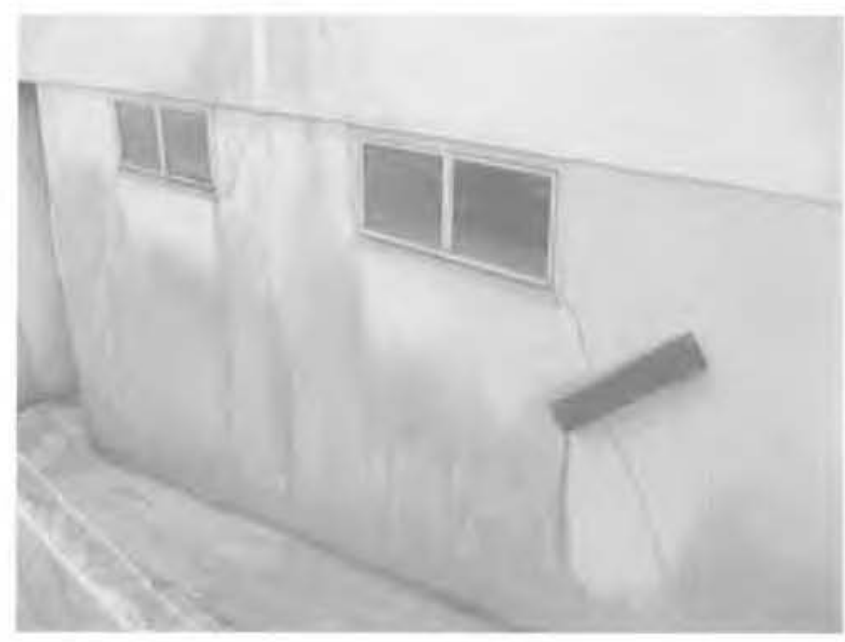

Fe. Vue sur fissure oblique (rampe du sous-sol bâtiment E) avec capot de protection de J'instrumentation.

View of disonder and instrumentation.

\section{Diagnostic}

Pour établir le diagnostic, la reconnaissarce a comporté la réalisation de huit sondages pressionétriques, de deux sondages de type Standard Penetration Test, ainsi que de qualire sondages carottés. Parallèlement, des puits de reconnaissance des fondations ont été exécutés pour caractériser leur nature et permettre le prélèvement et l'analyse en laboratoire des terrains d'assise. (Fig. 6).

Les sondages ont mis en évidence la présence de remblais sur $2 \mathrm{~m}$. d'épaisseur maximum puis les argiles à meultère reconnues au maximum jusqu'à $8,50 \mathrm{~m}$. de profondeur, peu à moyennement consistantes. Ces argiles, terrains d'assise des semelles de fondation (ancrage de 0,80 m par rapport au niveau du sous sol), ont montré par des essais en laboratoire, une teneur en eau naturelle sur les deux premiers mètres de l'ordre de 20 à $25 \%$ et une augmentation de près de 10 points à $3 \mathrm{~m}$ de profondeur. Les teneurs en eau généralement constatées pour cette formation sont voisines de $35 \%$, ce qui montre indéniablement le dessèchement en surface des argiles (Fig. 7). La détermination des pres. sions de gonflement sur cette même formation a mis en evidence des terrains avec une forte propension au gonflement avec des valeurs de l'ordre de $0,2 \mathrm{MPa}$ à $2 \mathrm{~m}$ de profondeur et de $0,5 \mathrm{MPa}$ à $4 \mathrm{~m}$ de profondeur. Les sables de Fontainebleau ont été reconnus directement sous les argiles à meulière jusqu'à 15,50 m de profondeur. Les essais effectués dans cette formation caractérisent des terrains de très bonne compacité. 
Résidence du Parc d'Ardenay 9, rue Bertaud - PALAISEAU

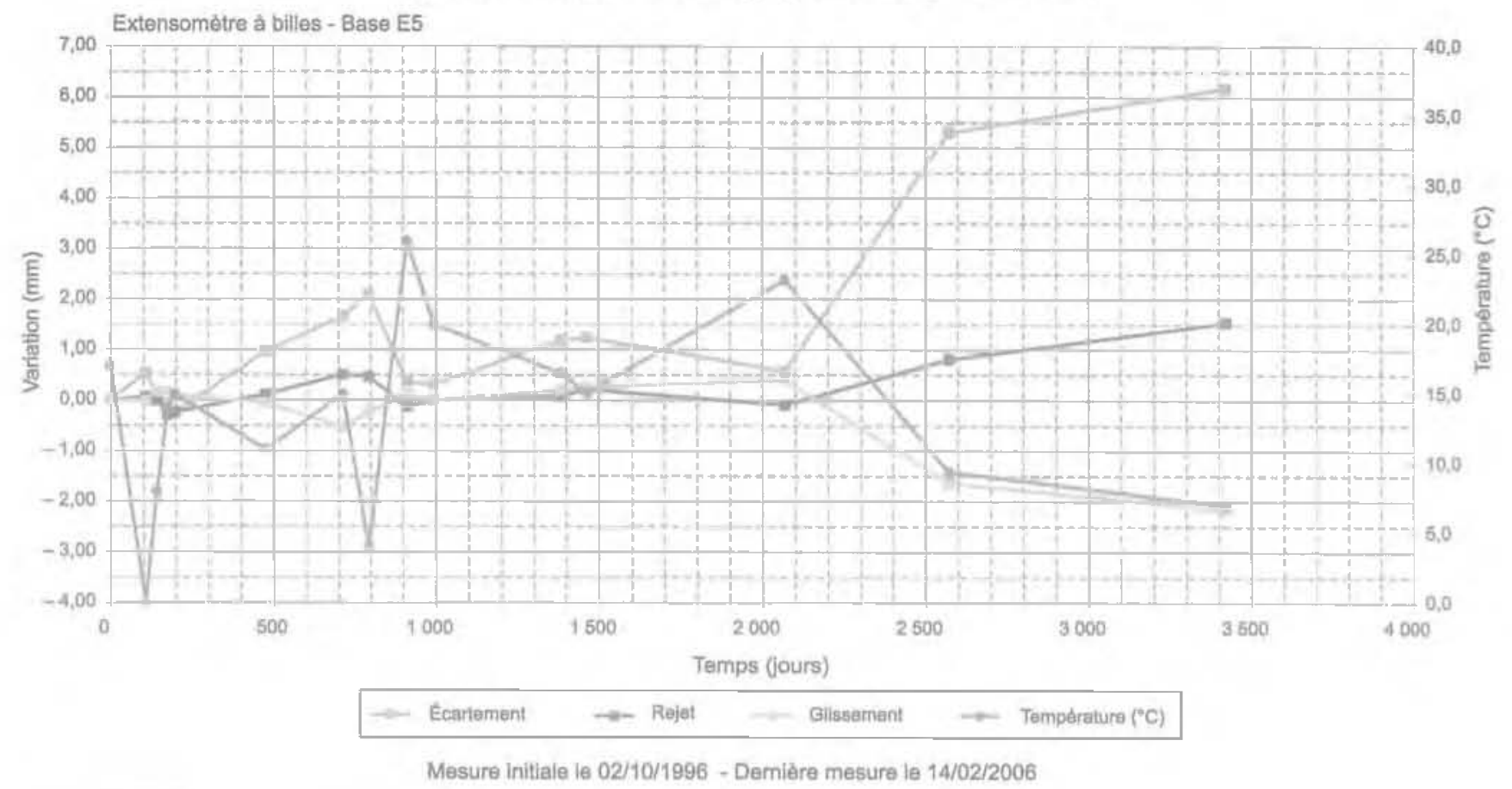

M. 3 Courbe de suivi des mesures d'ure nivele du battiment $\mathbf{E}$. Curve of measurements of instrumentation of the building $E$.

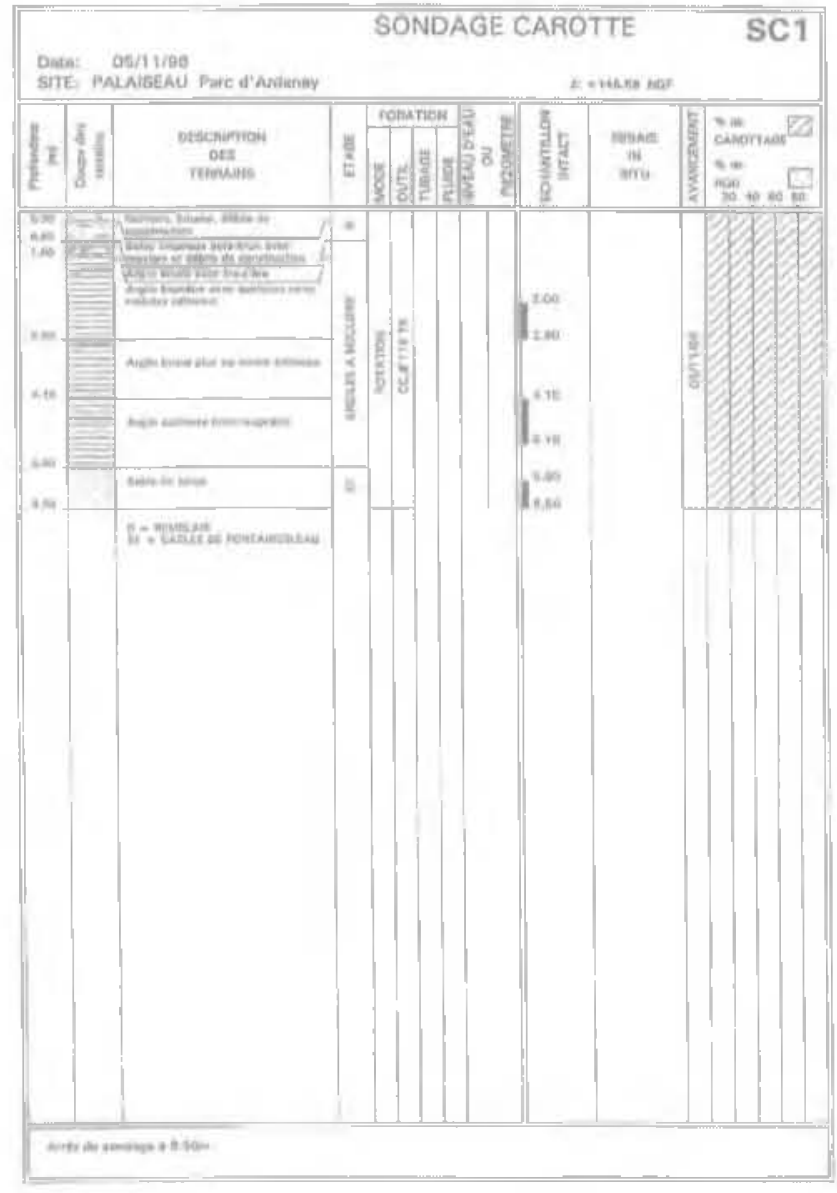

F3. - Sondage carotté réalisé au droit du bátiment $\mathrm{D}$ (doc. Arcadis). Sounding core on building D.
Teneur en eau w (\%)

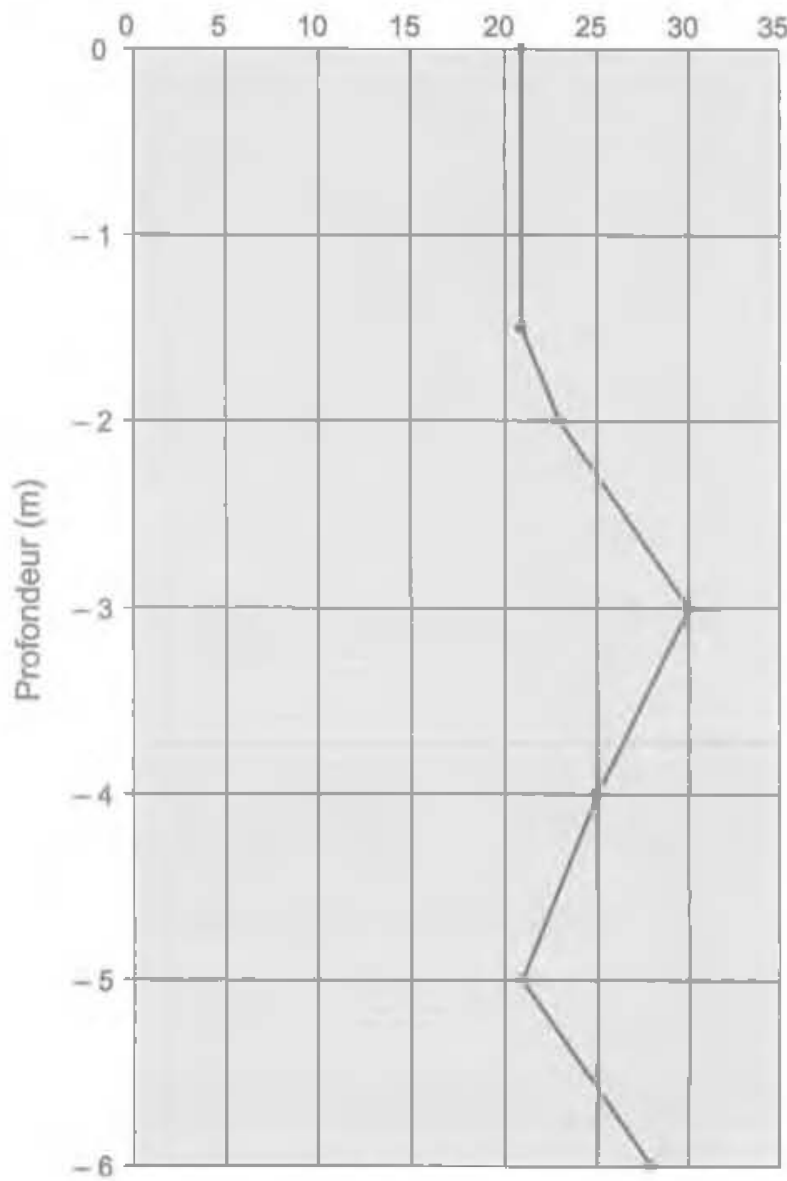

ac. 1 Profil hydrique des matériaux (doc. Arcadis\}.

Material's hydric section.. 
L'auscultation des deux bâttiments a mis en évidence des mouvements cycliques, d'amplitude variable d'un point à un autre, liés aux variations thermiques saisonnières. Les fissures ont tendance à s'ouvrir au cours de l'été el se refermer en hiver, toutefois les mouvements n'apparaissent pas toujours entiêrement réversibles. Ceci est caractéristique d'un sol d'assise avec une tendance à la dessiccation quí induit un tassement des fondations des deux bâtiments.

\section{4}

\section{La conception générale du disposi- tif de confortement}

\section{1}

\section{Renforcement de la structure}

Au vu des désordres qui affectent les deux immeubles et de leur évolution, if a été nécessaire de procéder au renforcement de la structure, celle-ci reposant en intérieur sur des appuis ponctuels de type poteaux et en périphérie sur des voiles; ces stmuctures étant fondées dans les argiles à meulière.

Le projet de confortement comprend un dispositif de longrines en béton armé liaisonnées aux fondations existantes et reposant sur des micropieux au droit des poteaux et des voiles périphériques. Ces longrines ont pour but de redonner une rigidité suffisante à l'immeuble, de manière à s'opposer à une nouvelle évolution localisée des tassements et/ou gonflement et à une évolution, de faible amplitude, de mouvements horizontaux.

Les reconnaissances géotechniques menées dans le cadre de l'expertise ont montré que le toit des formations insensibles aux variations de teneurs en eaux se situe vers $6 \mathrm{~m}$ de profondeur environ. Pour la pérennité du dispositif de longrines, il importe donc de reporter les charges amenées par les voiles et poteaux dans ces terrains, soit un ancrage entre $6 \mathrm{~m}$ et $14 \mathrm{~m}$ de profondeur, en fonction des descentes de charges, dans les sables de Fontainebleau.

Au total sont prévus 266 micropieux pour le bâtiment $D$ et 460 micropieux pour le bâtiment $E$, dimensionnés pour reprendre une charge de 90 tonnes maximum, au droit des poteaux les plus chargés, et une charge maximale de $30 \mathrm{~T} / \mathrm{ml}$ au droit des voiles béton. Ces charges importantes ont amené à la réalisation de micropieux de type III (avec injection sous pression) gui donnent un meilleur frottement latéral dans les terrains traversés et permettent ainsi une reprise de charge plus importante qu'un micropieu de type II.

\section{2}

\section{Phasage}

La chronologje prévue pour les travaux de chaque bâtiment (durée de l'ordre de 16 mois) est la suivante : - démolition des dallages et des murs relends des caves ;

- réalisation des micropieux;
- terrassement pour longrine et recépage des micropieux;

- liaisonnement des micropieux par les longrines pour les voiles ou massifs au droit des poteaux;

- remblaiement des fouilles et mise à niveau de la plateforme des sous-sols,

- réalisation du dallage ;

- réfection des murs de caves.

\section{3}

\section{Micropieux}

Les travaux de micropieux ont commencé après validation des hypothèses de dimensionnement au moyen d'essais de traction sur des micropleux réalisés en dehors de l'emprise des bâtiments. Les essais ont donc permis de valider la longueur de scellement des micropieux et de vérifier que les charges de rupture et de fluage ne sont pas atteintes dans les contraintes réelles du projet.

Les micropieux de type III ont été réalisés au moyen de deux ateliers par bâtiment, avec des machines équipées d'enregistrements en continus des paramètres de forage. Après traçage et implantation des micropieux, un carottage des fondations existantes a été effectué d'un diamètre de $175 \mathrm{~mm}$ pour les nicropieux de diamètre 150 , et de $220 \mathrm{~mm}$ pour les micropieux de diamètre 200. Le forage est effectué a l'aide d'un taillant perdu de diamètre $150 \mathrm{~mm}$ ou $200 \mathrm{~mm}$ avec mise en place en continu de barres métalliques autoforantes de diamètre extérieur de $73,5 \mathrm{~mm}$ pour les micropieux de $200 \mathrm{~mm}$ et de $49,3 \mathrm{~mm}$ pour les micropieux de diamètre $150 \mathrm{~mm}$. Pendant le forage des micropieux, un coulis de ciment dosé $1200 \mathrm{~kg} / \mathrm{m}^{3}$ est injecté en continu avec une pression de l'ordre de 10 bars. Le coulis est injecté à saturation et l'excédent est pompé, tamisé et recyclé dans la cuve d'injection. L'injection est maintenue à la profondeur prévue et jusqu'à la remontée de coulis propre. Après I'injection, les micropleux sont tubés sur toute la hauteur des argiles au moyen de tube PVC descendus à l'aide de la tête de forage afin de s'affranchir des frottements parasites induits par les éventuels gonflements et retraits de cette formation liés aux variations hydriques (Fig. 8).

\section{4}

\section{Longrines}

Les terrassements sont d'abord réalisés mécaniquement devant les voiles périphériques, jusqu'à l'assise des fondations. Au droit des micropieux des voiles, et par des passes de $1,5 \mathrm{~m}$ à $2 \mathrm{~m}$, les terrassements sont réalisés sous la fondation existante. Le micropieu est recépé à la hauteur prévue pour être liaisonné aux armatures de la longrine. (Fig. 9). Au droit de chaque poteau isolé, les têles de massifs sont scellées en contre-ceuve par l'intermédiàre d'armatures liaisonnées aux micropieux (Fig. 10 et 11). 


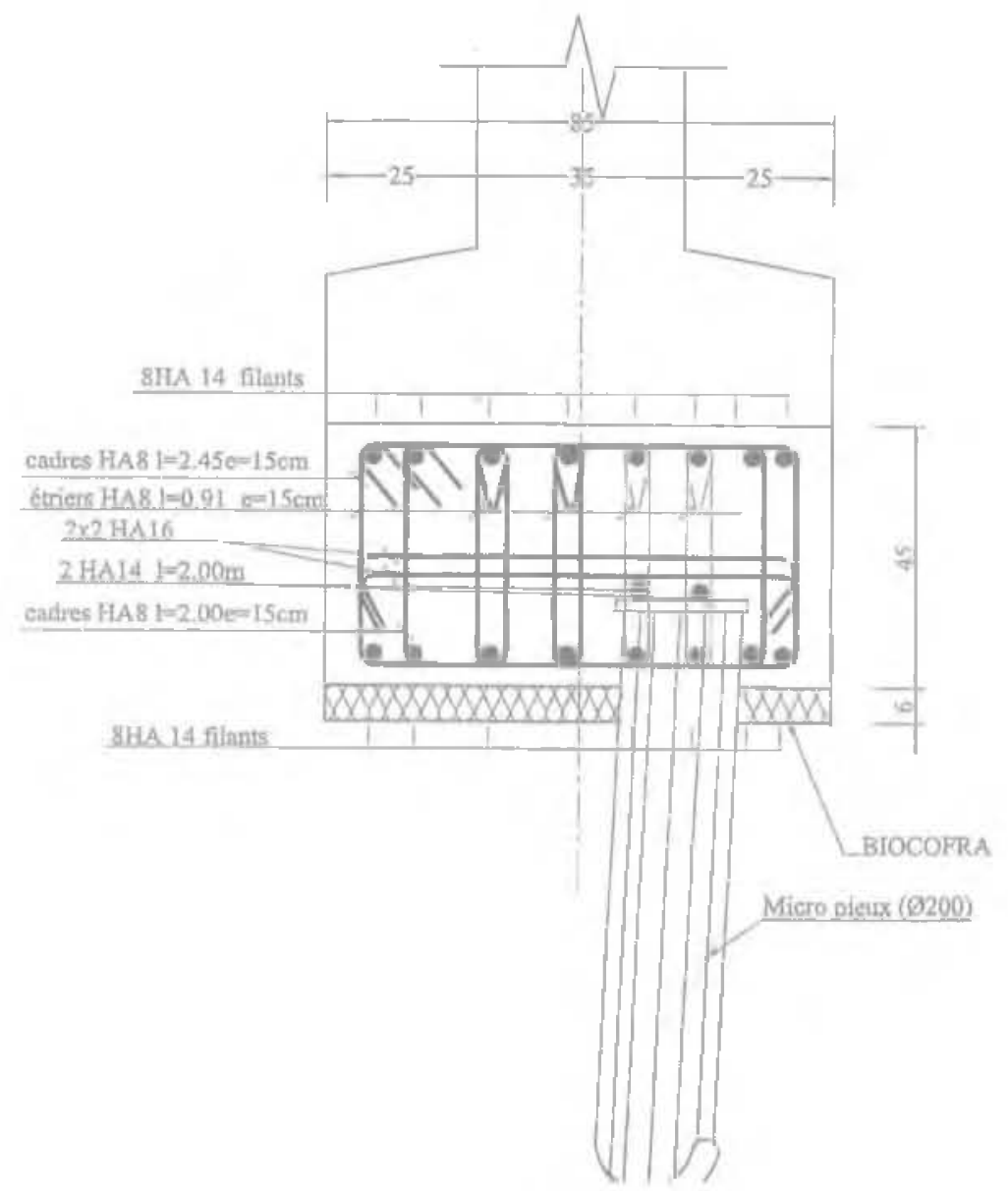

FG. Plan de principe de reprise des voiles par longrines au droit d'un micropieu (Cahier des clauses techniques et particulières, Cabinet Croué-Landaz, 2004).

Section on underpinning at level of micropile.

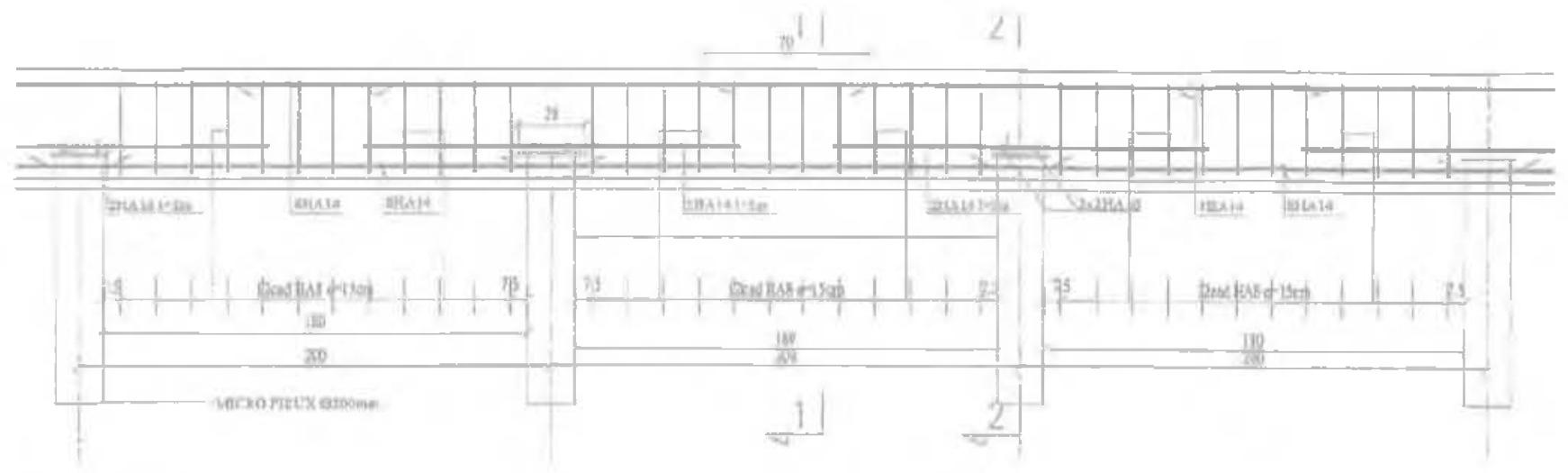

F5. Plan de principe de reprise par micropieux et longrine sous voile

(Cahier des clauses techniques et particulières, Cabinet Croué-Landaz, 2004)

Section on underpiruing by micropiles and footings. 


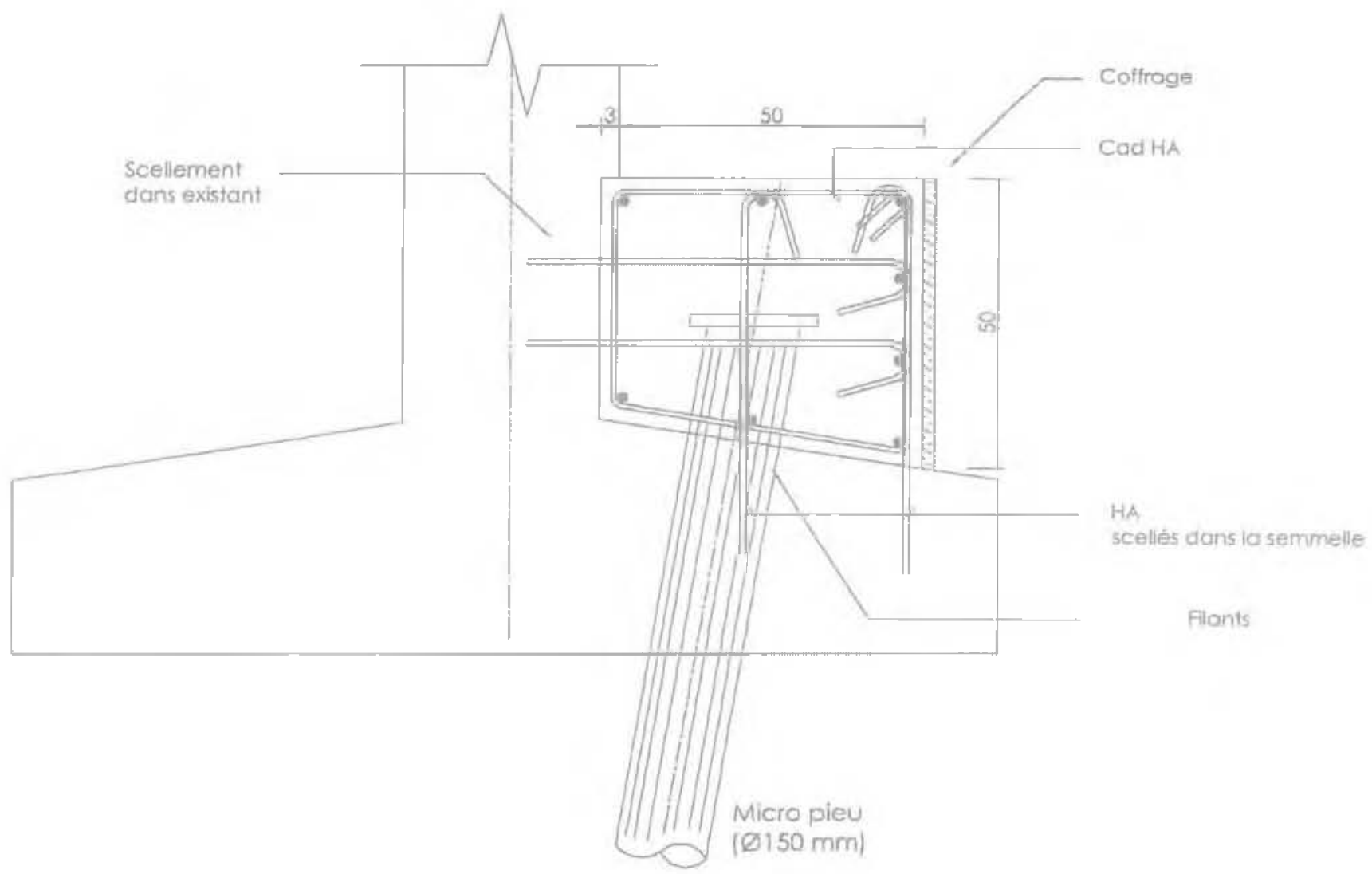

FIG. 10 Coupe de principe de coffrage et ferraillage sous poteau (Cahier des clauses techniques et particulières, Cabinet Croué-Landaz, 2004). Section on shuttering and reinforcement under bearn.

Les armatures, comprenant aciers filants et cadre de répartition sont de type HA18 et HA20 et sont réalisées directement dans la fouille sur un coffrage biodégradable. Ce coffrage permet grâce au viđe créé sous la longrine après sa dégradation, de s'affranchir des possibles gonflements des argiles en surface qui affecteraient les nouvelles fondations. Après la mise en place d'un coffrage en bois, le béton, dosé à $350 \mathrm{~kg} / \mathrm{m}^{3}$ est coulé pour englober les micropieux et les armatures et ainsi créer la nouvelle fondation.

\section{5}

\section{Travaux de remblais et dallage}

A la fin des travaux, les fouilles réalisées sont comblêes par les matériaux extraits et soigneusement compactés. La plate-forme est réalisée après la purge, la mise à niveau et le compactage du terrain sur lequel sera disposé un dallage béton. Pour ce dallage, réalisé sur terre-plenn et dissocié des voiles périphériques, des nappes de treillis soudés ont été disposées sur toute la superficie du sous-sol des bâtiments (Fig. 12). Le béton a été coulé pour atteindre une épaisseur de $15 \mathrm{~cm}$ environ avec l'exécution de joints de fractionnement, permettant en cas de gonflement des argiles sous-jacentes de prévenir l'apparition de fissures sur dallage.

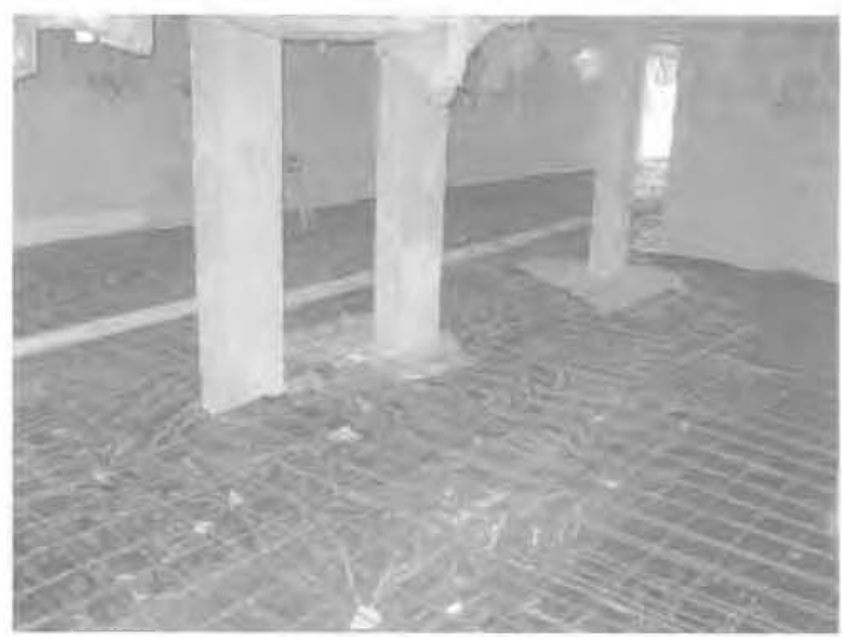

月å.1. Treillis soudé avant coulage du béton de dallage.

Girder before casting of pavement.

\section{Contrôle des exécutions}

Pendant toute la durée des travaux, les contrôles ont été effectués par des visites régulières sur site avec la 1 


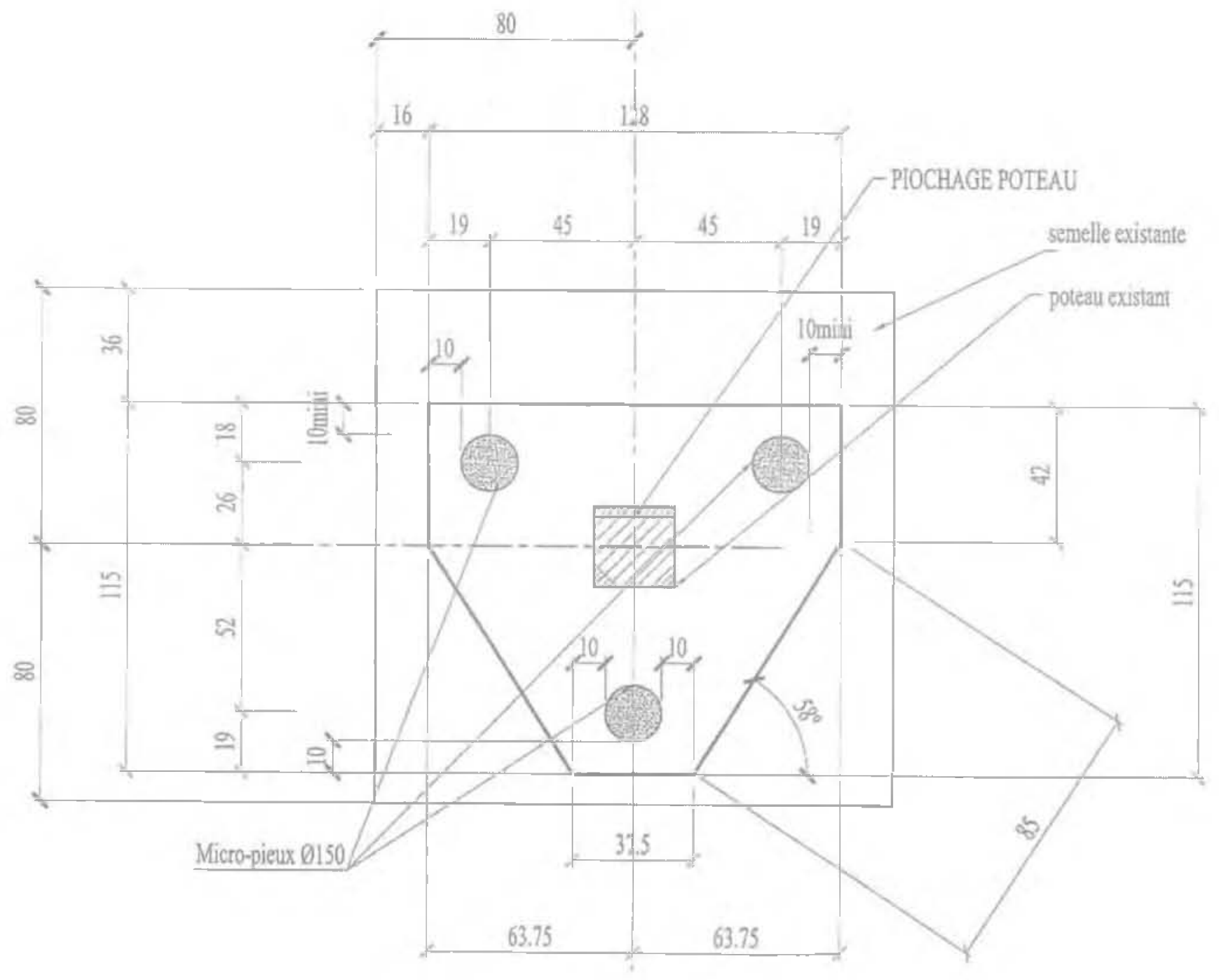

Fa. 11 Plan de principe de reprise des poteaux

(Cahier des clauses techniques et particulières, Cabinet Croué-Landaz, 2004).

Cabinet Crous-Landaz, 2004)

Plan view of undempining of beams.

vérification des matériaux utilisés et des méthodologies d'exécution mises en place. Cés contrôles ont notamment comporté, outre les essais de traction sur micropieux, des essais d'écrasement sur les coulis de micropieux.

Une fois les travaux de reprise en sous-øuvre complètement terminés, il est nécessaire de laisser un délai entre la fin des travaux et la rẻparation des fissures. Une période d'une année entière sera donc adoptée, de manière à ce que les charges des bâtiments se transfèrent dans les micropieux ce qui entraîne de nouveaux mouvements. Cette période révolue, il sera donc possible de reprendre toute la superstructure endomomagée dư bâtiment.

\section{$\overline{\text { Bibliographie }}$}

BRGM - Carte géologique de Corbeil à l'échelle de $1 / 50000,1979$.
Cabinet Croué-Landaz, Arcadis ESG-Cahjer des clauses techniques particulière de la reprise en sous-couve des bătiments B et D du parc d'Ardeney, 2004. 